\title{
Rigidity, Functional Equations and the Calogero-Moser Model
}

\author{
H. W. Braden* \\ Department of Mathematics and Statistics, \\ The University of Edinburgh, \\ Edinburgh, UK
}

\begin{abstract}
Suppose we have a natural Hamiltonian $H$ of $n$ particles on the line, centre of mass momentum $P$ and a further independent quantity $Q$, cubic in the momenta. If these are each $S_{n}$ invariant and mutually Poisson commute we have the Calogero-Moser system with potential $V=\frac{1}{6} \sum_{i \neq j} \wp\left(q_{i}-q_{j}\right)+$ const.
\end{abstract}

\section{Introduction}

The following note deals with many particle Hamiltonian systems on the line and their integrability. Although such systems arise in many physical settings and have been extensively studied there still is no simple way to determine their integrability or otherwise. General arguments [17] tell us that many particle Hamiltonian systems for sufficiently repulsive potentials are integrable, yet there appear few direct methods of actually solving for such systems. The integrable systems we can actually solve seem to form a very privileged class. The result presented here sheds some light on this state of affairs. We will follow a less well known route to the study of integrable systems, that employing functional equations.

Here we address the following question: What $S_{n}$ invariant, natural Hamiltonian systems of $n$-particles on the line and conserved centre of mass momentum admit a third independent, $S_{n}$ invariant, mutually Poisson commuting quantity, cubic in the momenta? (The precise statement and explanation of these terms will be given below.) Our answer is somewhat surprising. This data characterises the $a_{n}$ Calogero-Moser systems. This is the "rigidity" of our title. Although no restrictions were placed on further Poisson commuting invariants we arrive at a system for which sufficient exist to yield complete integrability. The mixture of symmetry and polynomial momentum is powerful. Such natural requirements and our result go some way in explaining the ubiquity of this class of models. The situation is somewhat reminiscent of the original work of Ruijsenaars and Schneider [28] who, when demanding certain commutation properties, discovered a class of Hamiltonian systems that proved to be integrable. It is also analogous to what one encounters with $\mathrm{W}$ and related algebras, where a few commutation relations specify the whole structure. Indeed, the connections between Conformal Field Theory and these models may mean this is more than analogy [23, 18 .

There are obvious generalisations to this work which will be taken up in the discussion. Before turning to the statement and proof of the result (given in the following two sections) it is perhaps worth making some general remarks on connections between integrable systems and functional equations. Functional equations have of course a long and interesting history in

*E-mail:hwb@ed.ac.uk 
connection with mathematical physics and touch upon many branches of mathematics [1], 21. They have arisen in the context of completely integrable systems in several different ways. We have already mentioned the mentioned the work of Ruijsenaars and Schneider. Hietarinta similarly derived a functional equation when seeking a second quartic integral for two particle systems on the line 19. A further way in which they arise is by assuming an ansatz for a Lax pair, the consistency of the Lax pair yielding functional and algebraic constraints. In this manner Calogero discovered the elliptic Calogero-Moser model [13] and Bruschi and Calogero constructed Lax pairs for the Ruijsenaars models [8, 9]. The functional equations found by this route appear 涵 as particular examples of

$$
\phi_{1}(x+y)=\frac{\left|\begin{array}{ll}
\phi_{2}(x) & \phi_{2}(y) \\
\phi_{3}(x) & \phi_{3}(y)
\end{array}\right|}{\left|\begin{array}{ll}
\phi_{4}(x) & \phi_{4}(y) \\
\phi_{5}(x) & \phi_{5}(y)
\end{array}\right|}
$$

The general analytic solution of this has been given by Braden and Buchstaber [5]. Interestingly, Novikov's school have shown that the Hirzebruch genera associated with the index theorems of known elliptic operators arise as solutions of functional equations which are particular examples of this. The string inspired Witten index was shown by Ochanine to be described by Hirzebruch's construction where now the elliptic function solutions were important [20]. A similar approach based upon an ansatz and consequent functional equations was used by Inozemtsev [21] to construct generalisations of the Calogero-Moser models, while in [6] this route was used to construct new solutions to the WDVV equations. Various functional equations have also arisen when studying the properties of wave-functions for associated quantum integrable problems. Gutkin found several functional relations by requiring a nondiffractive potential [16] while Calogero [14] and Sutherland [29, 30] obtained functional relations by seeking factorizable ground-state wave-functions. A recurring equation in this latter approach is

$$
\left|\begin{array}{ccc}
1 & 1 & 1 \\
f(x) & g(y) & h(z) \\
f^{\prime}(x) & g^{\prime}(y) & h^{\prime}(z)
\end{array}\right|=0, \quad x+y+z=0 .
$$

This finds general solution in [10, 3]. In our present work we will make use of the particular case of this equation [10, 3]:

Theorem 1 Let $f$ be a three-times differentiable function satisfying the functional equation

$$
\left|\begin{array}{ccc}
1 & 1 & 1 \\
f(x) & f(y) & f(z) \\
f^{\prime}(x) & f^{\prime}(y) & f^{\prime}(z)
\end{array}\right|=0, \quad x+y+z=0 .
$$

Up to the manifest invariance

$$
f(x) \rightarrow \alpha f(\delta x)+\beta,
$$

the solutions of (1) are one of $f(x)=\wp(x+d), f(x)=e^{x}$ or $f(x)=x$. Here $\wp$ is the Weierstrass $\wp$-function and $3 d$ is a lattice point of the $\wp$-function.

Perhaps one reason for the underlying connection between integrability and functional equations is that fact that Baker-Akhiezer functions obey such relations. Such connections between integrable functional equations and algebraic geometry have been studied by Buchstaber and Krichever [11] and Dubrovin, Fokas and Santini 15. Whatever, these connections between functional equations and complete integrability warrant further investigation. 


\section{The Result}

The result discovered is the following:

Theorem 2 Let $H$ and $P$ be the (natural) Hamiltonian and centre of mass momentum

$$
H=\frac{1}{2} \sum_{i=1}^{n} p_{i}^{2}+V, \quad P=\sum_{i=1}^{n} p_{i} .
$$

Denote by $Q$ an independent third order quantity

$$
Q=\sum_{i=1}^{n} p_{i}^{3}+\frac{1}{6} \sum_{i \neq j \neq k} d_{i j k} p_{i} p_{j} p_{k}+\sum_{i \neq j} d_{i j} p_{i}^{2} p_{j}+\frac{1}{2} \sum_{i j} a_{i j} p_{i} p_{j}+\sum_{i} b_{i} p_{i}+c .
$$

If these are $S_{n}$ invariant and Poisson commute,

$$
\{P, H\}=\{P, Q\}=\{Q, H\}=0,
$$

then $V=\frac{1}{6} \sum_{i \neq j} \wp\left(q_{i}-q_{j}\right)+$ const and we have the Calogero-Moser system.

Some explanatory remarks are in order. Here $S_{n}$ invariance means that for any coefficient $\alpha_{i j}\left(q_{1}, q_{2}, \ldots, q_{n}\right)$ in the expansions above we have $\alpha_{\sigma(i) \sigma(j)}\left(q_{\sigma(1)}, q_{\sigma(2)}, \ldots, q_{\sigma(n)}\right)$ for all $\sigma \in$ $S_{n}$. In particular $V\left(q_{1}, q_{2}, \ldots, q_{n}\right)=V\left(q_{\sigma(1)}, q_{\sigma(2)}, \ldots, q_{\sigma(n)}\right)$ for all $\sigma \in S_{n}$. We remark

that had we begun with particles of possibly different particle masses, $H=\frac{1}{2} \sum_{i=1}^{n} m_{i} p_{i}^{2}+V$, the effect of $S_{n}$ invariance is such as to require these masses to be the same. Thus we are assuming the $S_{n}$ invariant Hamiltonian of the introduction. Finally, by "an independent third order quantity" $Q$ we mean one functionally independent of $H$ and $P$ and for which one cannot obtain an invariant of lower degree by subtracting multiples of $P^{3}$ and $P H$. We are not dealing with quadratic conserved quantities here.

\section{The Proof}

Our proof has five steps. We begin by noting that the Poisson commutativity $\{Q, H\}=0$ yields (with $\left\{q_{i}, p_{j}\right\}=\delta_{i j}$ )

$$
\begin{aligned}
0= & \frac{1}{6} \sum_{l} \sum_{i \neq j \neq k}\left(\partial_{l} d_{i j k}\right) p_{i} p_{j} p_{k} p_{l}+\sum_{l} \sum_{i \neq j}\left(\partial_{l} d_{i j}\right) p_{i}^{2} p_{j} p_{l}+\sum_{i, j, l}\left(\partial_{l} a_{i j}\right) p_{i} p_{j} p_{l} \\
& +\sum_{i, j}\left(\partial_{i} b_{j}\right) p_{i} p_{j}-3 \sum_{i} p_{i}^{2}\left(\partial_{i} V\right)-\frac{1}{2} \sum_{i \neq j \neq k} d_{i j k}\left(\partial_{k} V\right) p_{i} p_{j} \\
& -\sum_{i \neq j} d_{i j}\left(2\left(\partial_{i} V\right) p_{i} p_{j}+\left(\partial_{j} V\right) p_{i}^{2}\right)-\sum_{i, j} a_{i j}\left(\partial_{i} V\right) p_{j}+\sum_{i}\left(\partial_{i} c\right) p_{i}-\sum_{i} b_{i} \partial_{i} V .
\end{aligned}
$$

The steps then are:

1. First we show that the $d_{i j k}$ and $d_{i j}$ terms in (3) may be taken to be zero.

Having made this simplification we then focus on the terms remaining in (雨) independent and quadratic in the momenta:

$$
\begin{aligned}
\partial_{j} b_{i}+\partial_{i} b_{j} & =0, \quad i \neq j, \\
\partial_{i} b_{i}-3 \partial_{i} V & =0 \\
\sum b_{i} \partial_{i} V & =0
\end{aligned}
$$


2. Second, using (5,6) we show that $b_{j}$ may be written in the form

$$
b_{j}=\sum_{i \neq j} W\left(q_{i}-q_{j}\right)+U\left(q_{j}\right),
$$

where $W$ is an even function.

3. Third, using $\{P, Q\}=0$, we may set $U=0$.

4. Fourth, that we may rewrite (7) in the form

$$
0=\sum_{i<j<k}\left|\begin{array}{ccc}
1 & 1 & 1 \\
W\left(q_{i}-q_{j}\right) & W\left(q_{j}-q_{k}\right) & W\left(q_{k}-q_{i}\right) \\
W^{\prime}\left(q_{i}-q_{j}\right) & W^{\prime}\left(q_{j}-q_{k}\right) & W^{\prime}\left(q_{k}-q_{i}\right)
\end{array}\right| .
$$

5. Finally we argue that each term in the sum (9) itself vanishes and so we arrive at an equation of the form (1). The result then follows simply.

Step 1. We begin by focusing on the terms in (任) quartic in the momenta. For $l$ different from $i, j, k$ we see that $\partial_{l} d_{i j k}=0$, and so $d_{i j k}=d_{i j k}\left(q_{i}, q_{j}, q_{k}\right)$. Further, from the coefficients of $p_{i}^{3} p_{j}, p_{i}^{2} p_{j}^{2}$ and $p_{i}^{2} p_{j} p_{k}$ (for $i, j, k$ distinct) respectively, we find

$$
\partial_{i} d_{i j}=0, \quad \partial_{j} d_{i j}=0, \quad \partial_{j} d_{i k}+\partial_{k} d_{i j}+\partial_{i} d_{i j k}=0 .
$$

The first and third of these together show $\partial_{i}^{2} d_{i j k}=0$ and so $d_{i j k}$ is at most linear in $q_{i}$. By symmetry

$$
d_{i j k}=\alpha q_{i} q_{j} q_{k}+\beta\left(q_{i} q_{j}+q_{j} q_{k}+q_{k} q_{i}\right)+\gamma\left(q_{i}+q_{j}+q_{k}\right)+\delta .
$$

Now using $\{P, Q\}=0$ shows $\alpha=\beta=\gamma=0$. Thus $d_{i j k}$ is a constant. This fact, together with the second and third equations of (10), shows $\partial_{k}^{2} d_{i j}=0$. Therefore $d_{i j}$ is at most linear in $q_{k}$ (for $k \neq i, j$ ). The first two equations of (10) show $d_{i j}$ is independent of $q_{i}$ and $q_{j}$. Now a similar argument employing $\{P, Q\}=0$ yields $d_{i j}$ to be constant. By subtracting appropriate multiples of $P^{3}$ and $P H$ we may then remove the $d$ terms from $Q$. Our assumption of independence means that the leading term of $Q$ does not vanish when doing this. Thus (after such a subtraction and a possible rescaling) we may set the $d$ terms in $Q$ to be zero. Henceforth we will assume this simplification has been made.

Step 2. Suppose $i, j, k$ are distinct. Then from (5) we obtain $\left(\partial_{i j}=\partial_{i} \partial_{j}\right.$ etc. $)$

$$
\partial_{j k} b_{i}+\partial_{i k} b_{j}=0, \quad \partial_{j k} b_{i}+\partial_{i j} b_{k}=0 .
$$

Taking the difference of these we see $\partial_{i}\left(\partial_{k} b_{j}-\partial_{j} b_{k}\right)=0$ and so

$$
-\partial_{k} b_{j}+\partial_{j} b_{k}=2 F\left(q_{j}, q_{k}\right) \text {. }
$$

Combining this with $\partial_{k} b_{j}+\partial_{j} b_{k}=0$ we obtain

$$
\partial_{j} b_{k}=F\left(q_{j}, q_{k}\right)=-F\left(q_{k}, q_{j}\right)=-\partial_{k} b_{j} .
$$

We wish to further restrict the form of $F$. If we apply $\partial_{i}$ to (5) and then use (6) we see

$$
-\partial_{i} \partial_{i} b_{j}=\partial_{i} \partial_{j} b_{i}=3 \partial_{i} \partial_{j} V=\partial_{j} \partial_{i} b_{j}
$$

and so

$$
\left(\partial_{i}+\partial_{j}\right) \partial_{i} b_{j}=0
$$


Therefore

$$
\partial_{i} b_{j}=F\left(q_{i}-q_{j}\right), \quad F(x)=-F(-x) .
$$

Upon integrating we obtain (8) where $W^{\prime}(x)=F(x)$ and $W$ is an even function. (In principle upon integrating the odd function $F$ we obtain a function $\widetilde{W}$ where $\widetilde{W}^{\prime}(x)=F(x)$ and $\widetilde{W}(x)=\widetilde{W}(-x)+\tilde{c}$. A priori we cannot argue that the integration constant $\tilde{c}$ vanishes if $\widetilde{W}(0)$ is not defined, as happens for singular potentials. However setting $W(x)=\frac{1}{2}(\widetilde{W}(x)+\widetilde{W}(-x))$ again yields (8) up to a constant, which at this stage may be incorporated into the arbitrary function $U$.) We have employed the $S_{n}$ symmetry throughout this step to identify each of the possibly different functions $F, W$ and $U$ arising from each pair as the same.

Step 3. Now upon employing $\{Q, P\}=0$ we see $\sum_{i=1}^{n} \partial_{i} b_{j}=0$. Using (8) we deduce that $\partial_{i} U\left(q_{i}\right)=0$ and so $U\left(q_{i}\right)$ is a constant. Such a constant may be removed altogether by subtracting an appropriate multiple of $P$ from $Q$, or simply incorporated into a redefinition of $W(x)$. Whatever, we may take $U=0$. Then

$$
b_{i}^{2}=\sum_{j \neq i} W^{2}\left(q_{j}-q_{i}\right)+2 \sum_{j \neq k \neq i} W\left(q_{j}-q_{i}\right) W\left(q_{k}-q_{i}\right) .
$$

Step 4. Now employing (6, (7) we see $0=\sum_{i} \partial_{i} b_{i}^{2}$. Using (12) we obtain

$$
\partial_{i} b_{i}^{2}=-2 \sum_{j \neq i} W\left(q_{j}-q_{i}\right) F\left(q_{j}-q_{i}\right)+2 \sum_{j \neq k \neq i} \partial_{i}\left(W\left(q_{j}-q_{i}\right) W\left(q_{k}-q_{i}\right)\right)
$$

When we sum this expression over $i$ the first term will vanish using oddness and evenness properties. Thus we arrive at

$$
0=\sum_{i \neq j \neq k} \partial_{i}\left(W\left(q_{j}-q_{i}\right) W\left(q_{k}-q_{i}\right)\right) .
$$

Define $A_{i j k}$ by

$$
A_{i j k}=\partial_{i}\left(W_{j i} W_{k i}\right)+\partial_{j}\left(W_{i j} W_{k j}\right)+\partial_{k}\left(W_{i k} W_{j k}\right)=\left|\begin{array}{ccc}
1 & 1 & 1 \\
W_{i j} & W_{j k} & W_{k i} \\
F_{i j} & F_{j k} & F_{k i}
\end{array}\right|,
$$

where we use the shorthand $W_{i j}=W\left(q_{i}-q_{j}\right)$. Then from the functional form of $W$ we know

$$
A_{i j k}=A_{j i k}=A_{j k i}=\Psi\left(q_{i}-q_{j}, q_{j}-q_{k}, q_{k}-q_{i}\right)
$$

and is fully symmetric in $i, j, k$. Thus

$$
0=\sum_{i<j<k} A_{i j k}=\sum_{i<j<k}\left|\begin{array}{ccc}
1 & 1 & 1 \\
W\left(q_{i}-q_{j}\right) & W\left(q_{j}-q_{k}\right) & W\left(q_{k}-q_{i}\right) \\
W^{\prime}\left(q_{i}-q_{j}\right) & W^{\prime}\left(q_{j}-q_{k}\right) & W^{\prime}\left(q_{k}-q_{i}\right)
\end{array}\right| .
$$

which is equation (9).

Step 5. We now wish to argue that $A_{i j k}=0$. If we apply $\partial_{i j k}$ to 14 we find that

$$
\partial_{i j k} A_{i j k}=0,
$$


as only one term in the sum depends on $i, j, k$. Thus $\partial_{j k} A_{i j k}$ is independent of $q_{i}$, and consequently due to the functional form (13) it must be a function of $q_{j}-q_{k}$ only. Therefore we must have

$$
\partial_{j k} A_{i j k}=B\left(q_{j}-q_{k}\right)
$$

and so, after integration and use of symmetry,

$$
A_{i j k}=E\left(q_{i}-q_{j}\right)+E\left(q_{j}-q_{k}\right)+E\left(q_{k}-q_{i}\right)
$$

(where $E(x)=E(-x)$ and $E^{\prime \prime}(x)=-B(x)$ ). We may therefore rewrite (14) as

$$
0=\sum_{i<j} E\left(q_{i}-q_{j}\right)
$$

Taking the partial derivative $\partial_{i j}$ of this expression then gives $\partial_{i j} E\left(q_{i}-q_{j}\right)=0$, as only this term depends on both $i$ and $j$. This, together with the evenness of $E$ tells us that $E$ is a constant. In conjunction with (15) we deduce $E=0$. That is $A_{i j k}=0$. Therefore for each distinct triple $i, j, k$

$$
0=\left|\begin{array}{ccc}
1 & 1 & 1 \\
W\left(q_{i}-q_{j}\right) & W\left(q_{j}-q_{k}\right) & W\left(q_{k}-q_{i}\right) \\
W^{\prime}\left(q_{i}-q_{j}\right) & W^{\prime}\left(q_{j}-q_{k}\right) & W^{\prime}\left(q_{k}-q_{i}\right)
\end{array}\right| .
$$

But this is none other than (1). The even solution of this is $W(x)=\wp(x)$, up to a constant. Finally, using (8) and (6) we obtain the stated conclusion.

\section{Discussion}

Our result may be interpreted as a rigidity theorem for the $a_{n}$ Calogero-Moser system and in part explains this models' ubiquity: demanding a cubic invariant together with $S_{n}$ invariance necessitates the model. A detailed scrutiny of our proof shows several generalisations possible. A natural generalisation is to replace the $S_{n}$ invariance with the invariance of a general Weyl group $W$ and make connection with the Calogero-Moser models associated to other root systems [24, 25]. Quite a bit is known about the quantum generalisations in this regard. Given a commutative ring $\mathcal{R}$ of $W$-invariant, holomorphic, differential operators, whose highest order terms generate $W$-invariant differential operators with constant coefficients, then the potential term for the Laplacian $\mathcal{H}$ (the quantum Hamiltonian) has Calogero-Moser potential appropriate to $W[27,26]$. Our result suggests something stronger may be possible: that the form of the potential may be dictated from just a few elements of $\mathcal{R}$. Taniguchi's results [31] are indicative of the rigidity of these models: if $\mathcal{H}$ is the quantum Hamiltonian just discussed, and $\mathcal{Q}_{1,2}$ are holomorphic (but not a priori $\mathrm{W}$-invariant) differential operators of appropriate degrees for which $\left[\mathcal{Q}_{1,2}, \mathcal{H}\right]=0$, then $\mathcal{Q}_{1,2} \in \mathcal{R}$ and consequently $\left[\mathcal{Q}_{1}, \mathcal{Q}_{2}\right]=0$. Interestingly in the present work we have employed a functional equation elsewhere encountered in the quantum regime.

A further generalisation of this work would be to replace the natural Hamiltonian structure of our theorem with (say) Hamiltonians of Ruijsenaars type. We remark in passing that there are still several unsolved functional equations surrounding this model. One might also seek to relax the full $S_{n}$ invariance imposed here. By so doing this will allow the Toda models. As shown by Inozemtsev [22], the Toda models arise as scaling limits of the Calogero-Moser model, the latter being the "generic" situation 四. It would be interesting to understand this in terms of the coadjoint descriptions of these models.

Though perhaps not obvious, this work arose from trying to understand models conjectured to be integrable (see for example [7]). Given a putative integrable Hamiltonian, what might the invariants look like? The present work tells us that for $S_{n}$ invariant systems not of CalogeroMoser type we should look for conserved quantities quartic and above in the momenta. 


\section{Acknowledgements}

I am grateful to A. Mironov, A. Marshakov and A. Morozov together with the Edinburgh Mathematical Physics group for their comments on this work which was begun under the support of a Royal Society Joint grant with the FSU. This work was presented at the Workshop on "Mathematical Methods of Regular Dynamics" dedicated to the 150-th anniversary of Sophie Kovalevski and I would thank I. Komarov for his remarks.

\section{References}

[1] J. Aczel, Lectures on Functional Equations and their Applications, Academic Press, N.Y. 1966.

[2] J. Aczel and J. Dhombres, Functional Equations in several variables, Cambridge University Press, 1989.

[3] H.W. Braden and J.G.B. Byatt-Smith, On a Functional Differential Equation of Determinantal Type, Bull. Lond. Math. Soc. 31, 463-470 (1999).

[4] H.W. Braden and V.M. Buchstaber, Integrable Systems with Pairwise Interactions and Functional Equations, Reviews in Mathematics and Mathematical Physics 10, 121-166 (1997).

[5] H.W. Braden and V.M. Buchstaber, The General Analytic Solution of a Functional Equation of Addition Type, SIAM J. Math. Anal. 28, 903-923 (1997).

[6] H.W. Braden, A. Marshakov, A. Mironov and A. Morozov, Seiberg-Witten theory for a non-trivial compactification from five to four dimensions, Physics Letters B448, 195-202 (1999).

[7] H. W. Braden, A. Marshakov, A. Mironov and A. Morozov, On Double-Elliptic Integrable Systems. 1. A Duality Argument for the case of SU(2), hep-th/9906240

[8] M. Bruschi and F. Calogero, The Lax Representation for an Integrable Class Of Relativistic Dynamical Systems, Commun. Math. Phys. 109, 481-492 (1987).

[9] M. Bruschi and F. Calogero, General Analytic Solution of Certain Functional Equations of Addition Type, SIAM J. Math. Anal. 21, 1019-1030 (1990).

[10] V.M. Buchstaber and A.M. Perelomov, On the functional equation related to the quantum three-body problem, Contemporary mathematical physics, Amer. Math. Soc. Transl. Ser. $2, \mathbf{1 7 5}, 15-34(1996)$.

[11] V.M. Buchstaber and I.M. Krichever, Vector Addition Theorems and Baker-Akhiezer Functions, Teor. Mat. Fiz. 94, 200-212 (1993).

[12] F. Calogero, Exactly solvable one-dimensional many-body problems, Lett. Nuovo Cimento (2) $13,411-416$ (1975).

[13] F. Calogero, On a functional equation connected with integrable many-body problems, Lett. Nuovo Cimento 16, 77-80 (1976).

[14] F. Calogero, One-dimensional many-body problems with pair interactions whose exact ground-state is of product type, Lett. Nuovo Cimento 13, 507 - 511 (1975). 
[15] B. A. Dubrovin, A. S. Fokas and P. M. Santini, Integrable Functional Equations and Algebraic Geometry, Duke Math. Journal 76, 645-668 (1994).

[16] Eugene Gutkin, Integrable Many-Body Problems and Functional Equations, J. Math. Anal. Appl. 133, 122-134 (1988).

[17] G. Fusco and W. M. Olivia, Integrability of a System of $N$ Electrons Subjected to Coulombian Interaction, J. Differential Equations 135, 16-40 (1997).

[18] Giovanni Felder and Alexander P. Veselov, Shift operators for the quantum CalogeroSutherland problems via Knizhnik-Zamolodchikov equation, Comm. Math. Phys. 160, 259273 (1994). 259-273.

[19] J. Hietarinta, Direct methods for the search of the second invariant, Phys. Reports 147, 87-154 (1987).

[20] F. Hirzebruch, Th. Berger and R. Jung, Manifolds and Modular Forms, Vieweg, Wiesbaden (1992).

[21] V. I. Inozemtsev, Lax Representation with Spectral Parameter on a Torus for Integrable Particle Systems, Lett. Math. Phys. 17, 11-17 (1989).

[22] V. I. Inozemtsev, The finite Toda lattices, Comm. Math. Phys. 121, 629-638 (1989).

[23] Atsushi Matsuo, Integrable connections related to zonal spherical functions, Invent. Math. 110, 95-121 (1992).

[24] M. A. Olshanetsky and A. M. Perelomov, Classical integrable finite-dimensional systems related to Lie algebras, Phys. Rep. 71, 313-400 (1981).

[25] M. A. Olshanetsky and A. M. Perelomov, Quantum integrable finite-dimensional systems related to Lie algebras, Phys. Rep. 94, 313-404 (1983).

[26] Hiroyuki Ochiai, Toshio Oshima and Hideko Sekiguchi, Hideko Commuting families of symmetric differential operators, Proc. Japan Acad. Ser. A Math. Sci. 70, $62-66$ (1994).

[27] Toshio Oshima and Hideko Sekiguchi, Commuting families of differential operators invariant under the action of a Weyl group, J. Math. Sci. Univ. Tokyo 2, 1-75 (1995).

[28] S.N.M. Ruijsenaars and H. Schneider, A new class of integrable systems and its relation to solitons, Ann. Phys. (NY) 170, 370-405 (1986).

[29] B. Sutherland, Exact ground-state wave function for a one-dimensional plasma, Phys. Rev. Lett. 34, 1083-1085 (1975).

[30] B. Sutherland, Exact results for a quantum many-body problem in one dimension, Phys. Rev. A4, 2019-2021 (1971), II Phys. Rev. A5, 1372-1376 (1972).

[31] Kenji Taniguchi, On uniqueness of commutative rings of Weyl group invariant differential operators, Publ. Res. Inst. Math. Sci. 33, 257-276 (1997). 